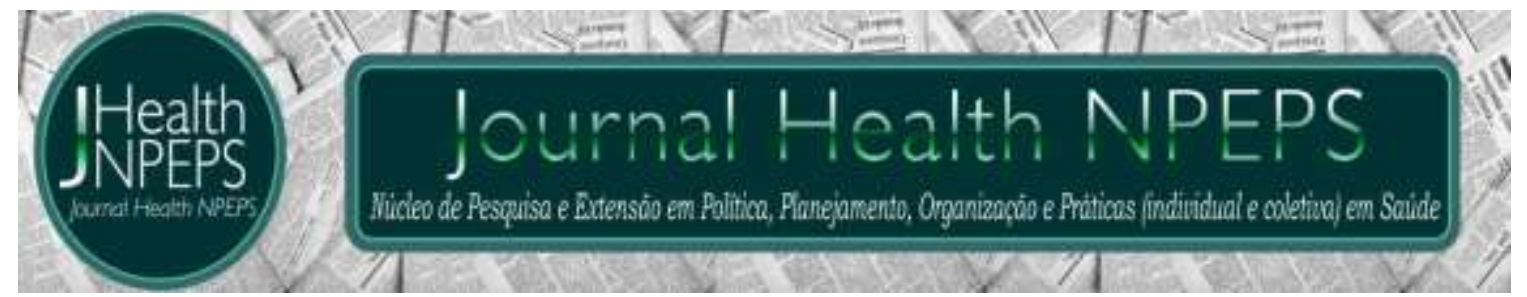

http://dx.doi.org/10.30681/252610103996

ARTIGO ORIGINAL

\title{
Efeitos da manobra de hiperinsuflação manual seguida da compressão descompressão torácica manual em pacientes oncológicos
}

\section{Effects of manual hyperinflation maneuver followed by manual chest compression and decompression in oncological patients}

\section{Efectos de maniobra de hiperinflación manual seguido de la compresión- descompresión torácica manual em pacientes oncológicos}

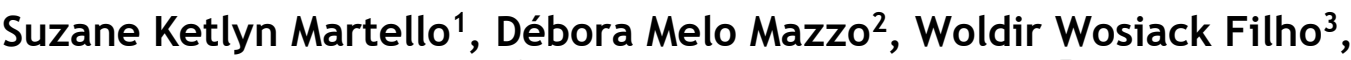 \\ Cesar Costa ${ }^{4}$, Juliana Carvalho Schleder ${ }^{5}$
}

\section{RESUMO}

Objetivo: avaliar os efeitos da manobra de hiperinsuflação manual (HM) isolada e associada à compressão descompressão torácica manual (CDTM) sobre a mecânica respiratória e os sinais vitais de pacientes oncológicos sob ventilação mecânica invasiva (VMI). Método: ensaio clínico não randomizado, com 23 pacientes oncológicos internados no Hospital Erasto Gaertner nos períodos de janeiro a junho de 2011 e agosto a dezembro de 2016, hemodinamicamente estáveis e sob VMI. Os dados de volume corrente (VC), volume minuto (Vmin), pressão de pico (Ppico), frequência cardíaca $(\mathrm{FC})$ e saturação periférica de oxigênio $\left(\mathrm{SpO}_{2}\right)$ foram avaliados antes da aplicação das manobras (AV1), imediatamente após a aplicação da HM (AV2) e 10 min após a aplicação da CDTM (AV3). Resultados: a $\mathrm{SpO}_{2}$ apresentou alteração significante estatisticamente entre a AV1 e AV2 $(p=0,010)$, cujas medianas foram 99 (96-100) e 100 (97-100), respectivamente, evolução atribuída à manobra de HM isolada. A Ppico e FC mantiveram-se

\footnotetext{
${ }^{1}$ Fisioterapeuta. Mestre em Tecnologia em Saúde. Hospital de Dermatologia Sanitária do Paraná. Piraquara, Paraná, Brasil. Email: susiketlyn@hotmail.com ORCID ID: https://orcid.org/0000-0003-4474-7045 Autor principal - Endereço para correspondência: Avenida Guatupê, 2484. Bairro Guatupê. Cidade São José dos Pinhais - Paraná, Brasil.

${ }^{2}$ Fisioterapeuta. Mestre em Ciências da Saúde. Hospital Universitário Regional dos Campos. Ponta Grossa, Paraná, Brasil. Email: deboramazzo@uol.com.br ORCID ID: https://orcid.org/0000-0002-1728-7648

${ }^{3}$ Fisioterapeuta. Mestre em Engenharia de Produção. Hospital Erasto Gaertner. Curitiba, Paraná, Brasil. Email: woldirwf@gmail.com ORCID ID: https://orcid.org/0000-0001-6593-7783

${ }^{4}$ Fisioterapeuta. Especialização em Fisioterapia Oncológica. Hospital Erasto Gaertner. Curitiba, Paraná, Brasil. Email: ccosta@terra.com.br ORCID ID: https://orcid.org/0000-0001-7222-5370

${ }^{5}$ Fisioterapeuta. Doutora em Fisiologia. Hospital Universitário Regional dos Campos. Ponta Grossa, Paraná, Brasil. Email: juschleder@yahoo.com.br ORCID ID: https://orcid.org/0000-0001-5789-7945
}

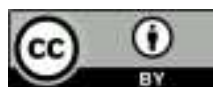

Este artigo está licenciado sob forma de uma licença Creative Commons Atribuição 4.0 Internacional, que permite uso irrestrito, distribuição e reprodução em qualquer meio, desde que a publicação original seja corretamente citada. 
estáveis. Conclusão: a associação da HM à CDTM não gerou alterações significativas sobre a mecânica respiratória e os sinais vitais de pacientes oncológicos sob VMI. Porém, a manobra de HM de forma isolada, mostrou-se eficaz na melhora da $\mathrm{SpO}_{2}$ nessa população, sem causar alterações hemodinâmicas significativas.

Descritores: Oncologia; Cuidados Críticos; Fisioterapia; Respiração Artificial.

\section{ABSTRACT}

Objective: to evaluate the effects of isolated manual hyperinflation (MH) maneuver and associated with manual chest compression and decompression (MCCD) on respiratory mechanics and vital signs of oncologic patients under invasive mechanical ventilation (IMV). Method: non-randomized clinical trial with 23 cancer patients admitted to the Erasto Gaertner Hospital, from January to June 2011 and August to December 2016, hemodynamically stable and under IMV. Data of tidal volume (TV), minute volume (MV), peak inspiratory pressure (Ppeak), heart rate $(\mathrm{HR})$ and peripheral oxygen saturation $\left(\mathrm{SpO}_{2}\right)$ were evaluated in three moments: before applying the maneuvers (AV1), immediately after the application of BS (AV2) and 10 min after application of MCCD (AV3). Results: the only variable that showed statistically significant change was $\mathrm{SpO}_{2}$ in the analysis between AV1 and AV2 ( $p=0.010)$, in which medians were 99 (96-100) and 100 (97-100), respectively, an evolution attributed to $\mathrm{MH}$ maneuver isolated. Ppeak and HR values remained stable. Conclusion: the association between $\mathrm{MH}$ manuever and MCCD did not generate changes on respiratory mechanics and vital signs of oncologic patients under IMV. However, the MH maneuver isolated was effective in improving $\mathrm{SpO}_{2}$ in this population without causing significant hemodynamic changes.

Descriptors: Medical Oncology; Critical Care; Physical Therapy Specialty; Respiration, Artificial.

\section{RESUMEN}

Introducción: evaluar los efectos de la maniobra de hiperinflación manual (HM) aislada y asociada con la compresión y descompresión torácica manual (CDTM) sobre la mecánica respiratoria y los signos vitales de pacientes oncologicos bajo ventilación mecánica invasiva (VMI). Método: ensayo clínico no aleatorio con 23 pacientes con cáncer ingresados en el Hospital Erasto Gaertner, de enero a junio de 2011 y agosto a diciembre de 2016, hemodinámicamente stables y baju VMI. Volumen corriente (VC), volumen minuto (Vmin), presión máxima (Ppeak), frecuencia cardíaca (FC) y saturación periférica de oxígeno $\left(\mathrm{SpO}_{2}\right)$ se evaluaron antes de la aplicación de las maniobras (AV1), inmediatamente después de la aplicación del HM (AV2) y 10 minutos después de la CDTM (AV3). Resultados: la $\mathrm{SpO}_{2}$ mostró un cambio estadísticamente significativo $(\mathrm{p}=0.010)$ entre AV1 y AV2, cuyas medianas fueron 99 (96-100) y 100 (97-100), respectivamente, evolución atribuida a la maniobra HM aislada. Ppeak y FC se mantuvieron estables. Conclusión: la asociación de la HM con CDTM no há generado diferencias significativas en la mecánica respiratoria y signos vitales de pacientes con cáncer bajo IMV. Sin embargo, la maniobra de MH solo fue efectiva para mejorar la $\mathrm{SpO}_{2}$ sin causar cambios hemodinámicos significativos. Descriptores: Oncología Médica; Cuidados Críticos; Fisioterapia; Respiración Artificial. 


\section{INTRODUÇÃO}

No decorrer do tratamento, o paciente oncológico está passível a ser internado em uma unidade de terapia intensiva (UTI), seja por influência de tratamentos mais agressivos, intervenções cirúrgicas ou por complicações da própria doença, o que requer, muitas vezes, a utilização de ventilação mecânica invasiva (VMI).

A VMI tem como objetivo principal promover as trocas gasosas, diminuir o consumo de oxigênio e, desta forma, o desconforto respiratório e o trabalho da musculatura respiratória, principalmente em situações de alta demanda metabólica. Além disso, permitir a aplicação de terapêuticas específicas ${ }^{1}$. Ainda que essencial, inclusive para a manutenção da vida, seu uso está sujeito a efeitos deletérios, como o acúmulo de secreções respiratórias, devido à tosse ineficaz em detrimento do não fechamento da glote, ocasionando prejuízo ao transporte do muco pela presença do tubo traqueal $^{2}$. Essa retenção de secreção contribui para episódios de hipoxemia, atelectasia ${ }^{3}$ e pneumonia associada à $\mathrm{VMI}^{4}$.

Neste contexto, o cuidado do profissional fisioterapeuta tem se mostrado eficaz e imprescindível nas $\mathrm{UTI}^{5}$. As principais metas desse cuidado no tratamento destas complicações incluem: higiene brônquica, reexpansão das áreas de atelectasia ${ }^{5,6}$ e aperfeiçoamento do mecanismo respiratório, visando a adequada oferta de $\mathrm{O}_{2}$ aos tecidos corporais e o equilíbrio ácidobásico ${ }^{1}$. Dentre as diversas técnicas e recursos utilizados para alcançar esses fins, duas manobras se destacam na prática cotidiana: a hiperinsuflação manual $(H M)$ e a compressão descompressão torácica manual (CDTM).

A HM também conhecida como Bag Squeezing (BS) consiste na hiperinsuflação pulmonar por meio de um reanimador manual, com liberação repentina da pressão no platô da hiperinsuflação, seguido de vibrocompressão realizada pelo fisioterapeuta durante a expiração e posterior remoção da secreção pela aspiração ${ }^{7}$. A técnica promove uma aceleração do fluxo expiratório, que gera fluxo turbulento e simula 
o mecanismo de tosse, além de potencializar as forças de recolhimento elástico pulmonar, favorecendo o deslocamento de secreção acumulada nas vias aéreas $^{8,9}$.

A manobra de CDTM consiste em uma compressão torácica, realizada manualmente pelo fisioterapeuta, direcionando os últimos arcos costais para dentro e para baixo durante a expiração, seguida de uma descompressão súbita no início da fase inspiratória. Essa variação dos fluxos inspiratório e expiratório favorece a desobstrução das vias aéreas e promove a reexpansão pulmonar.

Embora a maioria dos estudos compararem as técnicas isoladamente, a combinação entre duas ou mais técnicas é a prática mais utilizada por fisioterapeutas que atuam nas UTI5,10.

A investigação dos efeitos da associação da HM e CDTM poderá nortear os profissionais atuantes nos cuidados intensivos oncológicos e na eleição ou não dessas manobras durante 0 atendimento. Diante disso, o objetivo do estudo foi avaliar os efeitos da manobra de hiperinsuflação manual

(HM) isolada e associada à compressão descompressão torácica manual (CDTM) sobre a mecânica respiratória e os sinais vitais de pacientes oncológicos sob ventilação mecânica invasiva.

\section{MÉTODO}

Tratou-se de um ensaio clínico não randomizado, com abordagem quantitativa. Aprovado pelo Comitê de Ética em Pesquisa do Hospital Erasto Gaertner $\left(\mathrm{n}^{\circ}\right.$ 2066) situado em Curitiba-PR, e o consentimento livre e esclarecido foi obtido para todos os participantes, assinado pelo familiar responsável.

Os participantes da presente pesquisa foram recrutados entre pacientes oncológicos, independentemente do tipo de câncer, admitidos na UTI do Hospital Erasto Gaertner entre janeiro a junho de 2011 e agosto a dezembro de 2016, de acordo com os seguintes critérios de inclusão: ter idade igual ou superior a 18 anos; estar em tratamento fisioterapêutico; estar sedado com grau maior ou igual a 2 na Escala de 
Ramsay ${ }^{11}$ e estar sob VMI. Foram considerados critérios de exclusão: apresentar instabilidade hemodinâmica12; trauma ou lesão lítica em tórax; hemorragia peri/intraventricular e edema cerebral sob monitorização da pressão intracraniana (PIC).

Primeiramente, foi realizada a avaliação inicial (AV1) na qual foram coletados os seguintes dados: frequência cardíaca (FC), pressão arterial média (PAM), saturação periférica de oxigênio $\left(\mathrm{SpO}_{2}\right)$; volume corrente expirado (VC), pressão de pico inspiratório (Ppico) e volume minuto (Vmin). Previamente à aplicação das técnicas, foi ofertada uma fração inspirada de $\mathrm{O}_{2}\left(\mathrm{FiO}_{2}\right)$ de $100 \%$ durante dois minutos, ajustada no próprio ventilador mecânico, seguido da instilação de $3 \mathrm{ml}$ de soro fisiológico a 0,9\% na cânula endotraqueal. Em seguida, foi aplicada a manobra de HM por dois fisioterapeutas (F1 e F2). Para tanto, o F1 realizava uma hiperinsuflação com o uso de um reanimador manual do tipo $\mathrm{AMBU}^{\circledR}$ conectado a um fluxômetro de $\mathrm{O}_{2}$ a $10 \mathrm{~L} / \mathrm{min}$, mantendo comprimido ao final da inspiração, gerando uma pausa inspiratória de um a dois segundos. Durante a expiração, o F2 aplicava a manobra de vibrocompressão torácico manual bilateral. Após a aplicação da manobra, por cinco ciclos respiratórios, o F1 realizava a higiene brônquica por meio da aspiração das vias aéreas via cânula endotraqueal e o participante era reconectado ao ventilador mecânico, com os mesmos parâmetros anteriores à aplicação da técnica. Em seguida, o participante era reavaliado (AV2) da mesma forma como na avaliação inicial, a fim de avaliar os efeitos da manobra de HM de forma isolada.

Após a AV2, o $F 1$ dava sequência no protocolo, aplicando a manobra de CDTM, na qual era realizada uma compressão no início da expiração seguida de uma descompressão abrupta no início da inspiração subsequente. Repetiu-se a aplicação da técnica sete vezes em cada hemitórax e sete vezes de forma bilateral. Após 10 minutos da aplicação da CDTM foi realizada a avaliação final (AV3), da mesma forma como na AV1 e AV2, a fim de que as variáveis hemodinâmicas 
retornassem aos seus valores basais $^{2}$.

A análise estatística dos dados foi realizada por meio do software IBM SPSS Statistics (v. 21.0). A normalidade da distribuição das variáveis foi testada por meio do Teste de Shapiro-Wilk, considerando um nível de significância de $5 \%$ $(a=0,05)$. A FC foi a única variável cuja distribuição foi considerada normal, por isso optou-se por descrever os dados por meio de mediana e primeiro e terceiro quartil (Q1 - Q3), por serem mais representativos para distribuições não normais do que valores de média e desvio padrão.

0 teste não paramétrico Análise de Variâncias de Friedman de Amostras Relacionadas foi aplicado para comparar a diferença das variáveis nos três momentos de avaliação (AV1, AV2 e AV3). A análise Post Hoc foi seguida apenas para a $\mathrm{SpO}_{2}$, que foi a única variável que apresentou diferença significante estatisticamente entre os três momentos. Para tanto, foi aplicado o teste não paramétrico de Wilcoxon para as comparações dos três momentos de avaliação, dois a dois (AV1 x AV2; AV1 x AV3; AV2 x AV3), a fim de identificar entre quais momentos as diferenças foram significantes estatisticamente. Para essas análises foi realizada a Correção de Bonferroni (a/3) e o nível de significância assumido foi de 0,017.

\section{RESULTADOS}

Foram incluídos no estudo 26 participantes, dos quais três foram excluídos devido à instabilidade hemodinâmica, ficando a amostra constituída por 23 pacientes, 9 homens e 14 mulheres, com idade média de 57,5 $\pm 12,4$ anos. O Quadro 1 apresenta - diagnóstico clínico dos participantes. 
Quadro 1 - Diagnóstico clínico dos participantes do estudo.

\begin{tabular}{|c|c|}
\hline Participante & Diagnóstico Clínico \\
\hline 01 & Linfoma de célula T linfoblástico \\
\hline 02 & Adenocarcinoma de endométrio \\
\hline 03 & Meningeoma \\
\hline 04 & Carcinoma espinocelular de esôfago \\
\hline 05 & Tumor pâncreas \\
\hline 06 & Tumor ósseo \\
\hline 07 & Tumor do Sistema Nervoso Central \\
\hline 08 & Carcinoma espinocelular de seio piriforme \\
\hline 09 & Nódulo pulmonar - em investigação diagnóstica \\
\hline 10 & Mielofibrose \\
\hline 11 & Mielodisplasia \\
\hline 12 & Adenocarcinoma de próstata \\
\hline 13 & Icterícia - em investigação diagnóstica \\
\hline 14 & Anemia aplásica - em investigação diagnóstica \\
\hline 15 & Carcinoma espinocelular de colo uterino \\
\hline 16 & Adenocarcinoma de cárdia \\
\hline 17 & Hemangioblastoma cerebelar \\
\hline 18 & Leucemia mielóide aguda \\
\hline 19 & Melanoma \\
\hline 20 & Mieloma múltiplo \\
\hline 21 & Carcinoma ductal infiltrante de mama \\
\hline 22 & Carcinoma espinocelular de colo uterino \\
\hline 23 & Linfoma difuso de grandes células B \\
\hline
\end{tabular}

Tabela 1 - Comparações das variáveis nos três momentos de avaliação.

\begin{tabular}{lcccc}
\hline \multicolumn{1}{c}{ Variável } & AV 1 & AV 2 & AV 3 & $p$ \\
\hline VC $(\mathrm{ml})$ & $423(386-538)$ & $454(407-569)$ & $460(384-577)$ & 0,087 \\
Vmin $(\mathrm{L} / \mathrm{min})$ & $9,5(7,6-10,8)$ & $9,4(8,0-10,9)$ & $8,7(7,8-11,0)$ & 0,519 \\
Ppico $\left(\mathrm{cmH}_{2} \mathrm{O}\right)$ & $20(19-28)$ & $20(19-28)$ & $20(19-25)$ & 0,457 \\
$\mathrm{FC}(\mathrm{bpm})$ & $99(76-113)$ & $95(80-123)$ & $97(81-116)$ & 0,906 \\
$\mathrm{SpO}_{2}(\%)$ & $99(96-100)$ & $100(97-100)$ & $100(95-100)$ & $0,033^{*}$
\end{tabular}

VC: volume corrente; Vmin: volume minuto; Ppico: pressão de pico; FC: frequência 
cardíaca; $\mathrm{SpO}_{2}$ : saturação periférica de oxigênio. Valores de mediana (Q1 - Q3). Teste Análise de Variâncias de Friedman de Amostras Relacionadas. *Significância estatística $(a=0,05)$.

Na Tabela 1 estão apresentadas as comparações entre os três momentos de avaliação, com os valores de mediana (Q1-Q3) de todas as variáveis analisadas, com os respectivos valores de $p$ das comparações entre as avaliações.

Conforme observado na Tabela 1, o VC aumentou após a aplicação da manobra, o Vmin diminuiu, a Ppico permaneceu inalterada e a FC reduziu, porém em nenhuma destas variáveis houve alteração estatisticamente significativa. $\mathrm{A} \mathrm{SpO}_{2}$ aumentou e foi a única variável cuja diferença entre os três momentos de avaliação foi considerada significante. A análise Post Hoc para essa variável, mostrou diferença estatisticamente significativa entre as avaliações 1 e $2(p=0,010)$.

\section{DISCUSSÃO}

Conforme pode ser
observado pela análise das
amplitudes interquartílicas na
Tabela 1, o VC apresentou uma
elevação entre a AV1 e AV2, e se

manteve estável entre a AV2 e AV3. Diminuiu na comparação da AV1 com a $\mathrm{AV} 3$, porém em nenhuma comparação a diferença foi considerada significante estatisticamente. Da mesma forma, o Vmin não apresentou alterações significativas nas três comparações realizadas, cujo comportamento foi similar ao VC.

O estudo de Via, Oliveira e Dragosavac $^{13}$ investigou os efeitos da aplicação de manobras de higiene brônquica seguida da CDTM sobre o volume pulmonar de indivíduos em VMI, em modo de ventilação espontâneo e constatou um aumento significativo no VC e Vmin. Um aumento nos valores dessas variáveis também era esperado no presente estudo, visto que as manobras aplicadas incrementam 0 volume corrente pela hiperinsuflação pulmonar. Uma justificativa para tais achados pode estar relacionada ao fato dos participantes estarem sob VMI controlada, na qual a pressão inspiratória e frequência respiratória mantem-se constantes 
durante a maior parte dos ciclos respiratórios.

A Ppico permaneceu estável durante os três momentos de avaliação, cuja mediana manteve em $20 \mathrm{cmH}_{2} \mathrm{O}$. No estudo de Hodgson et $a l^{14}$, 0 alcance de pressões de pico de $40 \mathrm{cmH}_{2} \mathrm{O}$ durante as manobras de $\mathrm{HM}$ promoveu aumento na complacência estática e na depuração do muco. Corroborando com esses achados, Lemes et al ${ }^{15}$ observaram também aumento na complacência pulmonar ao atingir os mesmos valores de Ppico.

No presente estudo, não foi mensurada a Ppico atingida durante a aplicação das técnicas (apenas após), sendo uma sugestão para os próximos estudos. Porém, a estabilidade observada nos valores de Ppico permite julgar a técnica como segura, visto que pressões de pico muito elevadas podem causar lesões na barreira alvéolo-capilar ${ }^{16}$. No estudo de Ortiz et $\mathrm{al}^{17}$, os valores de pressão platô durante a aplicação da manobra de HM mantiveram-se dentro dos limites considerados seguros na prevenção de barotrauma. A pressão platô não foi avaliada no presente estudo, assim como a resistência e a complacência pulmonar, pois alguns participantes tinham drive respiratório, impossibilitando a coleta desses dados de forma adequada.

Com relação à FC, não foram observadas alterações significativas entre os três momentos de avaliação. Lobo, Cavalcante e Mont'Alverne ${ }^{18}$ compararam os efeitos da manobra de HM e da manobra de zeep (MZ) sob algumas variáveis cardiorrespiratórias, antes, durante e logo após as aplicações, em 20 pacientes sob VMI. Os autores observaram uma diferença significativa na FC apenas no grupo que recebeu a $\mathrm{HM}$, entre antes e durante e antes e após a aplicação da manobra, cujos valores apresentaram uma elevação progressiva, diferentemente dos resultados encontrados na presente pesquisa. No estudo de Dias et $a l^{2}$, os valores de FC aumentaram significativamente durante a realização da aspiração isolada e da HM com compressão torácica e aspiração, retornando aos valores basais após 5 min. A contradição nos resultados encontrados na 
literatura em relação às alterações hemodinâmicas de tais técnicas indica a necessidade de maiores pesquisas para chegar a conclusões mais seguras a esse respeito.

A falta de padronização da manobra de $\mathrm{HM}^{19}$, o tipo de ressuscitador usado e o próprio profissional fisioterapeuta que está aplicando a técnica contribuem para explicar a variabilidade nos desfechos encontrados na literatura, pois o VC depende do tamanho e tipo de balão auto inflável utilizado, se o fisioterapeuta utiliza uma ou duas mãos e do tempo de insuflação do balão 2,17,20. Com relação à manobra de CDTM, esta também depende da força exercida pelo fisioterapeuta durante a aplicação da compressão no tórax, apresentando-se como mais uma limitação na comparação entre os estudos.

A única variável que apresentou alteração significativa entre os momentos de avaliação foi a $\mathrm{SpO}_{2}$. Na análise Post Hoc, verificou-se um aumento significante estatisticamente entre a AV1 e AV2, devido provavelmente aos efeitos da hiperinsuflação na promoção da reexpansão de áreas colapsadas, favorecendo a circulação colateral e a difusão do $\mathrm{O}_{2}$ para a corrente sanguínea ${ }^{16}$. A falta de significância entre a AV1 e AV3 faz com que o efeito observado sobre a $\mathrm{SpO}_{2}$ seja atribuído à manobra de HM e não à associação com a CDTM. Corroborando com os resultados do presente estudo, pesquisadores ${ }^{21}$ também observaram um aumento significativo na $\quad \mathrm{SpO}_{2}$ imediatamente após a aplicação da HM em 20 pacientes sob VMI. De forma contraditória, pesquisa ${ }^{18}$ encontrou uma diminuição significativa nos valores de $\mathrm{SpO}_{2}$ logo após a aplicação da manobra. Os autores justificaram os achados pela perda da pressão positiva expiratória final (PEEP) durante a realização da HM.

A associação das manobras de HM e CDTM já é uma prática rotineira por parte de profissionais que atuam em terapia intensiva, e embora não tenha sido observada alterações significativas nos volumes pulmonares, não há evidências sólidas para rejeitar essa prática. Para isso, novos estudos devem ser realizados, estratificando a amostra de acordo 
com o tipo de câncer ${ }^{22}$ e topografia da lesão, e com avaliação de variáveis adicionais como, a pressão de platô, complacência, resistência e dados gasométricos.

\section{CONCLUSÃO}

Não foram observados benefícios significantes da associação da manobra de hiperinsuflação manual à manobra de compressão descompressão torácica manual sobre a mecânica ventilatória de pacientes oncológicos sob VMI. Apesar disso, a aplicação isolada da manobra de hiperinsuflação manual, mostrou-se efetiva no incremento da $\mathrm{SpO}_{2}$ nessa população, sem causar alterações hemodinâmicas importantes, sendo indicada para pacientes oncológicos com quadro de dessaturação.

\section{REFERÊNCIAS}

1. Carvalho CRR, Junior CT, Franca SA. III Consenso Brasileiro de Ventilação Mecânica Ventilação mecânica: princípios, análise gráfica e modalidades ventilatórias. J Bras Pneumol.
2007; 33(2):54-70.

2. Dias $C M$, Siqueira $T M$, Faccio TR, Gontijo LC, Salge JASB, Volpe MS. Efetividade e segurança da técnica de higiene brônquica: hiperinsuflação manual com compressão torácica. Rev Bras Ter Intensiva. 2011; 23(2):190-8.

3. Naue WS, Forgiarini Junior LA, Dias AS, Vieira SRR. Chest compression with a higher level of pressure support ventilation: effects on secretion removal, hemodynamics, and respiratory mechanics in patients on mechanical ventilation. J Bras Pneumol. 2014; 40(1):55-60.

4. Ntoumenopoulos $G$, Presneill $J$, McElholum M, Cade J. Chest physiotherapy for the prevention of ventilatorassociated pneumonia. Intensive Care Med. 2002; 28(7):850-6.

5. Jerre CG, Thelso R, Silva DJ, Beraldo MA, Gastaldi PA, Kondo C, et al. III CBVM Fisioterapia no paciente sob ventilação mecânica. J Bras 
Pneumol. 2007; 33(Supl 2):142-50.

6. Stiller K. Physiotherapy in intensive care: an updated systematic review. Chest. 2000; 118(6):1801-13.

7. Windsor $\mathrm{H}$, Harrison $\mathrm{G}$, Nicholson $\quad$ T. "Bag squeezing":

a physiotherapeutic technique. Med J Aust. 1972; 2(1):82932.

8. Moreira MR, Padilha KG. Ocorrências latrogênicas com Pacientes Submetidos à Ventilação Mecânica em Unidade de Terapia Intensiva. Acta Paul Enferm. 2001; 14(2):10-8.

9. Oliveira ABF, Dias OM, Mello $M M$, Araújo S, Dragosavac D, Nucci A, et al. Fatores associados à maior mortalidade e tempo de internação prolongado em uma unidade de terapia intensiva de adultos. Rev Bras Ter Intensiva. 2010; 22(3):250-6.

10. Martins RHG, Dias NH, Braz JRC, Castilho EC. Complicações das vias aéreas relacionadas à intubação endotraqueal. Rev Bras Otorrinolaringol. 2004; 70(5):671-7.

11. Ramsay MAE, Savege TM, Simpson BRJ, Goodwin R. Hospital Topics Controlled Sedation with AlphaxaloneAlphadolone. Br Med J. 1974; 22:656-9.

12. Margareth S, Lobo A, Rezende E, Mendes CL. Suporte Hemodinâmico. Consenso Bras Monit e Suporte Hemodinâmico - Parte V. 2006; 18:161-76.

13. Via FD, Oliveira RARA, Dragosavac D. Effects of manual chest compression and descompression maneuver on lung volumes, capnography and pulse oximetry in patients receiving mechanical ventilation. Brazilian J Phys Ther. 2012; 16(5):354-9.

14. Hodgson C, Denehy L, Ntoumenopoulos G, Santamaria J, Carroll S. An investigation of the early effects of manual lung hyperinflation in critically ill patients. Anaesth Intensive Care. 2000; 28(3):255-61. 
15. Lemes DA, Alvitos K, Sixel B.

Hiperinsuflação manual: Mechanical ventilator as a revisão de evidências physical therapy device in intensive care unit. Eur Respir J. 2006; 28(50):730.

16. Lemes DAS, Guimarães FS. 0 Uso da Hiperinsuflação como Recurso Fisioterapêutico em Unidade de Terapia Intensiva. Rev Bras Ter Intensiva. 2007; 19(2):221-225.

17. Ortiz TA, Forti G, Volpe MS, Carvalho CRR, Amato MBP, Tucci MR. Estudo experimental sobre a eficiência e segurança da manobra de hiperinsuflação manual como técnica de remoção de secreção. J Bras Pneumol. 2013; 39(2):205-13.

18. Cavalcante LA, Mont'Alverne DGB. Applicability of bag squeezing and zeep maneuvers in mechanically ventilated patients. Rev Bras Ter intensiva. 2010; 22(2):186-91.

19. Nunes GS, Botelho GV, Schivinski CIS.

Conflito de interesses: Os autores declaram não haver conflito de interesses.

- Concepção: Martello SK, Wosiack Filho W, Costa C, Schleder JC, 
Martello SK, Mazzo DM, Wosiack Filho W, Costa C, Schleder JC. Efeitos da manobra de hiperinsuflação...

- Desenvolvimento: Martello SK, Mazzo DM, Wosiack Filho W, Costa C, Schleder JC.

- Redação e revisão: Martello SK, Mazzo DM, Wosiack Filho W, Costa C, Schleder JC.

Como citar este artigo: Martello SK, Mazzo DM, Wosiack Filho W, Costa C, Schleder JC. Efeitos da manobra de hiperinsuflação manual seguida da compressão descompressão torácica manual em pacientes oncológicos. J Health NPEPS. 2020; 5(1):276-289.

Submissão: 07/11/2019

Aceito: $13 / 03 / 2020$

Publicado: $01 / 06 / 2020$ 